\title{
林木细根寿命及其影响因子研究进展
}

\author{
梅 $\quad$ 莉 ${ }^{1} \quad$ 王政权 ${ }^{1 *} \quad$ 程云环 ${ }^{1} \quad$ 郭大立 ${ }^{2}$ \\ （1 东北林业大学林学院, 哈尔滨 150040) （2 美国 J. W. Jones 生态学研究中心, Georgia 39870)
}

\begin{abstract}
摘 要 细根周转要消耗大量的 $\mathrm{C}$, 它影响森林生态系统 $\mathrm{C}$ 分配格局与过程和养分循环, 对生态系统生产力具有 重要意义。细根的周转取决于细根的寿命 细根寿命越短, 周转越快, 根系对 $\mathrm{C}$ 的消耗也越多。大量研究表明, 细 根的寿命与地上部分 $\mathrm{C}$ 向根系供应的多少有密切关系, 同时也与细根直径大小、土壤中 $\mathrm{N}$ 和水分的有效性、土壤温 度以及根际周围的土壤动物和微生物的活动有关。本文综述了国外近年来在该领域里的研究进展, 特别是对控制 细根寿命的机理和主要影响因子进行了评述, 目的是引起国内研究者的关注, 促进我国根系生态学的研究与发展。

关键词 森林 细根寿命 细根周转 根系 C 和 $\mathrm{N}$ 根系结构
\end{abstract}

\section{A REVIEW : FACTORS INFLUENCING FINE ROOT LONGEVITY IN FOREST ECOSYSTEMS}

\author{
MEI Li ${ }^{1}$ WANG Zheng-Quan ${ }^{*} \quad$ CHENG Yun-Huan ${ }^{1}$ and Guo Da-Li ${ }^{2}$ \\ (1 Forestry College , Northeast Forestry University , Harbin 150040 , China) \\ (2 Joseph W. Jones Ecological Research Center, Newton, Georgia 39870 , USA)
}

\begin{abstract}
Fine root longevity is an essential component of root ecology and physiology. It is also a critical determinant of root turnover and therefore carbon $(\mathrm{C})$ and nutrient flow in terrestrial ecosystems. Despite the ecological importance of fine root longevity and the tremendous research efforts devoted to it , the understanding of fine root longevity and turnover is still rudimentary. This article reviews some of the most important factors that control fine root longevity, including carbon allocation, fine root structure, soil nitrogen $(\mathrm{N})$ and water availability , soil temperature, and soil biota in forest ecosystems, with the purpose of providing a brief summary of recent advances in fine root longevity research and to point out the gaps of understanding and directions for future root longevity research in China.
\end{abstract}

The most important function of fine roots is resource acquisition from the soil. To perform this function , $\mathrm{C}$ fixed in leaves must be used to build the fine root biomass and to supply energy needed for root growth, resource uptake , and maintenance of the fine roots. Consequently , $\mathrm{C}$ availability to roots may exert strong control over root longevity, and the interactions between $\mathrm{C}$ source (leaves) and $\mathrm{C}$ sink (roots) has been postulated as a mechanism through which root longevity may be explained. However, due to the lack of experimental evidence, the mechanisms by which $\mathrm{C}$ is allocated to roots and how $\mathrm{C}$ availability in roots controls root longevity remains poorly understood. Detailed studies on $\mathrm{C}$ allocation and utilization in processes such as root growth and root respiration (in growth and maintenance) are needed.

In addition to physiological controls of root longevity (e.g. , C availability), structural characteristics of root systems also have a strong influence over root longevity. Recent studies showed that both root diameter and branching order were important regulators having shorter lifespans and turn over more rapidly than larger diameter, higher order roots. These findings are likely to contribute greatly to a more accurate quantification and prediction of $\mathrm{C}$ and nutrient flow via root turnover.

The efficiency model, which suggests that the mortality of fine roots may occur when the $\mathrm{C}$ costs of plant roots exceed the benefits (e.g. , nutrient acquisition) they provide, also has been invoked to explain root mortality and longevity. This model necessitates that root longevity is closely related to resource availability in the soil. Some evidence suggests that roots in resource rich sites live longer, while others suggest the opposite. Much of the controversy may result from different methods used in estimating fine root longevity and turnover , and differences in $\mathrm{C}$ sink-source relationships among different species, stand development, as well as the length of the experiments in which the influence of resource levels on root longevity are tested.

Climatic factors also influence root longevity. Fine root longevity appears to be the longest in cold environments with marked seasonal variations in seasonal environments. However, most recent evidence suggests that root longevity may be more dependent on root structure than on root environment, with first order roots having similar longevity regardless of differences in species and the root environment. 
Finally , ecosystem-scale processes, such as environmental stresses and pathogen and herbivory pressure , may also influence root longevity. Drought and high temperatures may shorten root longevity. However, because soil moisture , temperature, leaf C fixation , and soil resource availability are tightly linked , independent tests of moisture and temperature influences on root longevity are difficult to conduct. Evaluating the regulation of herbivores and pathogens on root longevity is also difficult, in part because little quantitative information is available on the populations of root pathogens and herbivores in different ecosystems and the responses of root systems to different levels of pathogen and herbivory pressure.

In sum, root longevity is a critical but difficult research subject. There are many opportunities as well as challenges for future root longevity research in China, of which we list only a few :1) C allocation and C utilization in fine roots may be a primary mechanism controlling root longevity , but , to understand this mechanism fully, the methods for accurately estimating $\mathrm{C}$ allocation and utilization in roots of different structural and functional characteristics must be developed first ;2) Root longevity is clearly related to soil resource availability (e.g. , $\mathrm{N}$ and moisture) and environmental factors (e.g. , soil temperature and moisture) , and further studies on the impact of these factors on the longevity of individual roots and root systems as a whole may prove fruitful. Studies in this area should be large-scale and long-term to encompass large environmental variability frequently observed in natural ecosystems and to provide relevant information for a better understanding of the belowground aspect of global environment change ; and , 3) Linking root structure, function , and longevity is likely to ce one of the most promising areas of root longevity research in the near future. Current technologies seem to be reasonably reliable for making estimates of root longevity on roots of different structural and functional features ; the main challenge lies in the time and effort needed to collect such information , particularly in a country as vast and diverse as China.

Key words Forests, Fine root longevity, Fine root turnover, Root carbon and nitrogen , Root structure

细根 (直径 $\leqslant 2 \mathrm{~mm}$ ) 是植物吸收养分和水分的 主要器官, 其寿命是指从出生到死亡的这段时间, 是 细根重要的生理生态学特性。细根的生长、衰老、死 亡、脱落和再生长过程称为细根周转 (Vogt et al., 1986)，该过程要消耗大量的 C ( Hendricks et al., 1993 ; Bloomfield et al. , 1996 ; Eissenstat \& Yanai, 1997)和释放出养分 (Vogt et al. , 1986)。在大多数 陆地生态系统中，细根的生物量虽然只占地下总生 物量的 3\% 30\%左右, 但由于细根处于周转过程 之中，维持这个动态过程要消耗净初级生产力的 10\% 75\% 左右 (Vogt et al . , 1986 ; Raich \& Nadelhoffer , 1989 ; Gill \& Jackson , 2000)。因此 细根周转 与生态系统生产力有密切关系 (Hendricks et al. , 2000 ; Nadelhoffer, 2000)。通过细根周转而归还到 土壤中的 C 和养分超过地上凋落物 (Raich \& Nadelhoffer，1989; 黄建辉等,1999; 张小全等,2000), 是生 态系统物质循环和能量流动的重要组成部分, 近年 来已成为生态系统 $\mathrm{C}$ 分配格局与过程研究的核心 环节 (Nadelhoffer , 2000 ; Gill \& Jackson ,2000)。

细根周转归根结底是由细根寿命决定的 (Schoettle \& Fahey, 1994)。细根的寿命越短, 周转越 迅速 (Bloomfield et al. , 1996 ; Eissenstat \& Yanai , 1997)，对 C 的消耗也越大 (Hendrick \& Pregitzer， 1993 ; Steele et al., 1997 ; Gill \& Jackson , 2000) ; 从 另一方面来讲, 归还到林地的养分越多, 养分及 $\mathrm{C}$ 的循环也加速 (Jackson et al. , 1997 ; 李凌浩等,
1998)。细根的寿命长则几年或数月，短则几天，生 长在不同的立地上的同一树种的细根寿命有较大的 差异, 甚至不同季节长出的细根寿命也不相同 (Eissenstat \& Yanai , 1997 ;张小全等,2001)。究竟哪些因 素控制着细根的寿命? 这个问题一直是细根寿命研 究的重点(Eissenstat et al. 2000)。为此, 近些年来 国内外生态学者分别从影响根系的生物因素和非生 物因素, 如光合产物的分配过程 (Farrar \& Jones, 2000)、细根直径大小和分枝方式(Wells \& Eissenstat， 2001 ; Pregitzer et al . , 1997;2002)、土壤 $\mathrm{N}$ 和水分的 有效性 (Norby \& Jackson，2000)、土壤温度 (Pregitzer et al. , 2000)、根际微生物 (Bloomfield et al. , 1996) 以及研究方法 (Eissenstat \& Yanai, 1997) 等方面进行 了深入研究。本文对影响细根寿命的几个重要因素 的研究概况和进展进行介绍, 以便引起我国相关研 究人员的重视, 促进我国根系研究的深入。

\section{1 细根寿命与 $\mathbf{C}$ 消耗}

根系在树木体内有 源” 和 汇” 的双重功能, 是 养分、水分的 源”和 “ 汇” (Farrar \& Jones , 2000 ; Anderson, 2003)。细根与其它器官一样也有其生活 史。细根寿命受树木体内 C 源-C 汇分配关系的控 制, 分配到细根的 C 主要用于 4 个方面, 即细根伸 长、呼吸及养分吸收与同化、有机物分泌、土壤植食 动物 (Herbivory)等(Chapin III et al. ,2002)。一些证 据表明, 细根吸收养分和水分越多, 分配到细根的 C 也就越多 (Bloomfield et al. , 1996 ; Hendricks et al. ， 
1997）其寿命也较长。一旦细根周围养分耗尽, 吸 收能力减弱, C 向细根分配立刻减少 (Burton et al., 2000)，细根则衰老(Pregitzer et al ., 1993 ; Eissenstat \& Yanai，1997 ; Majdi，2001)进而死亡。Högberg 等 (2001) 在欧洲赤松 (Pinus sylvestris) 林分做环割试 验, 证明 C 向细根分配减少后, 根系呼吸明显降低, 其原因可能是细根衰老和死亡增加。

树木体内关于 $\mathrm{C}$ 向细根分配过程并通过该过 程控制细根寿命的机理还不清楚, 根系研究者分别 从细根环境和细根功能方面提出不同假设。针对细 根环境方面,Hendricks 等 (1993) 根据细根养分吸收 依赖于 $\mathrm{C}$ 的投入，对其控制机理给出了两种假设 :1) 异化分配 (Differential allocation) ,即随 $\mathrm{N}$ 在系统中的 有效性的提高, 分配给细根的 C 的相对比例下降， 细根的寿命或死亡率保持不变。2)恒定分配 (Constant allocation），即随 $\mathrm{N}$ 有效性的提高，分配给细根 的 $\mathrm{C}$ 的比例保持不变, 根系死亡率增加。在针叶林 中连续钻取土芯法的研究结果多支持异化分配假设 ( Comeau \& Kimmins, 1989 ; Gower et al., 1992 ; Haynes \& Gower, 1995)。在落叶林中使用 C-N 平衡 法的研究结果多支持恒定分配假设 (Nadelhoffer et al., 1985 ; Raich \& Nadelhoffer, 1989 ; Hendricks et $a l$. , 1993)。在细根功能方面,Farrar 和 Jones (2000)给 出了 4 种假说 :1)功能平衡假说, 即根系得到的 $\mathrm{C}$ 量 取决于根系与叶之间的功能平衡关系 ,2) 推力假说 (Push) 即根系的 C 取决于地上部分 C 的输入 3) 拉 力假说 (Pull) 即根系的 C 获取是自身的一种功能, 分 配到根系中 $\mathrm{C}$ 的多少受根内对 $\mathrm{C}$ 需求所控制 4 )共同 控制假说 即分配到根系的 C 受地上部分和地下部分 因子的共同控制。 $\mathrm{C}$ 的减少引起细根衰老和生命终 结是一个复杂的生理生态过程 (Farrar \& Jones , 2000 ; Anderson , 2003) 难点在于影响光合产物 C 分配的内 部和外部因素还不清楚, 目前仅是通过 源-汇”关系 去解释。野外实验研究表明, 上述理论和假说并不能 完全解释 C 控制细根寿命的机理, 主要是树种与环境 的多样性 (Farrar \& Jones , 2000 ; Nadelhoffer, 2000)。 一些假说都是针对某一具体的生态系统提出的, 是否 具有普适性, 还有待于检验。尽管如此, 我们认为 $\mathrm{C}$ 向地下分配的多少是影响细根寿命的重要因素。

\section{2 细根的寿命与细根形态}

直径是根系形态的重要指标之一, 对寿命有很
大的影响 (Eissenstat \& Yanai, 1997)。直径越细的 根, N 的浓度较高 (Gordon \& Jackson, 2000) , 非木质 化程度高 (Hendricks et al . 2000) , 寿命较短 (Eissenstat \& Yanai , 1997)。在 60 年生的欧亚械 (Acer pseudoplatanus) 林中, 直径 $<0.25 \mathrm{~mm}$ 细根的 $(\mathrm{N}$ 浓度 24.4 $\mathrm{mg} \mathrm{g^{-1 }}$ )平均寿命 $<300 \mathrm{~d}$, 而直径 $>0.25 \mathrm{~mm}$ 细根的 $\left(\mathrm{N}\right.$ 浓度 $13.0 \mathrm{mg} \mathrm{g}^{-1}$ )平均寿命则 $>600 \mathrm{~d}$ ( Pregitzer et al. , 1997)。Wells 等 (2002) 对 15 年生的碧桃 (Prunus persica) 细根存活分析表明, 直径 $\leqslant 0.25 \mathrm{~mm}$ 的细根寿命 $140 \mathrm{~d}$, 直径为 $0.25 \sim 0.5 \mathrm{~mm}$ 的细根寿 命 $262 \mathrm{~d}$, 而直径 $>0.5 \mathrm{~mm}$ 的细根寿命 $426 \mathrm{~d}$ 左右。 我国鼎湖山南亚热带森林直径 $\leqslant 2 \mathrm{~mm}$ 的细根平均 寿命 $630 \mathrm{~d}$, 直径 $2 \sim 5 \mathrm{~mm}$ 的细根寿命 $1050 \mathrm{~d}$ 左右 (温达志等,1999)。

根系生长过程中产生复杂分枝系统, 形成不同 等级的根序(Pregitzer et al. , 1997)。根序的分级依 赖于根系分枝的起源 (Fitter，1985)，根系最先端的 细根为一级根 (起源于二级细根)、上推二级细根 (起 源于三级细根)、三级细根 (起源于四级细根) 等 (Pregitzer et al. , 2002)。根序对细根寿命的影响与 直径类似，N的浓度随根序降低而增加，C 的浓度则 有不同程度的减少 (Pregitzer et al.，1997；Majdi， 2001 ; Wells et al. ,2002)。因此,一级细根(常常非 木质化) 其寿命较短, 根序较高的细根寿命较长。 Pregitzer 等 (2002) 对北美 4 种阔叶树和 5 种针叶树 研究表明, 直径小于 $0.5 \mathrm{~mm}$ 的细根根长占直径小于 $1.5 \mathrm{~mm}$ 根长的 $80 \%$ 左右。根序等级与直径正相关， 与 $\mathrm{N}$ 浓度负相关 (Pregitzer et al. , $1997 ; 2002$ )。基 于 Pregitzer 等 $(1997 ; 2002)$ 发现，细根死亡的顺序可 能与发育顺序相反, 一级根可能先死, 随后是二级 根, 再后是三级根、四级根等。Wells 等 (2002) 用两 年的时间对 15 年生碧桃的研究表明，二级和三级细 根平均寿命 $452 \mathrm{~d}$, 而一级细根的平均寿命为 $190 \mathrm{~d}$ 左右。我们认为从根系发育的角度来讲, 研究不同 根序的寿命问题可能更合理，因为对于某些树种，在 直径小于 $0.5 \mathrm{~mm}$ 的细根中, 既包括一级细根又包括 二级和三级细根，但是它们的寿命是不同的。然而， 一些研究数据表明, 在最细的前三个根序当中, 长叶 松 (Pinus palustris) 同一级根序的细根在直径方面的 变异往往大于 $0.3 \mathrm{~mm}^{1)}$ 。最近的研究表明, 在木本 植物中, 无论种类和根系环境如何, 根系的结构 (尤 其是分枝方式) 可能是与寿命有关的一个最基本 
特征(Ruess et al .,2003)。但是, 直径和根序如何独 立地影响细根寿命还是一个尚待解决的问题, 主要 是细根死亡的过程和顺序不清楚。我们认为随着估 计根系寿命方法的可靠性相对提高, 如微根管法 (Minirhizotron)、同位素法 (Isotope)、尤其是各种方法 联合使用等, 这为揭示根系结构对根系寿命调控机 理的研究提供了保障。

\section{3 细根寿命与土壤 $\mathbf{N}$ 的有效性}

$\mathrm{N}$ 是林木生长需求量最多的限制性养分，根系 获取 $N$ 包括吸收、运输及同化 3 个过程。吸收 $\mathrm{N}$ 需 要消耗大量的 C (Eissenstat et al. , 2000 ; Norby \& Jackson，2000)。投入到细根中 C 的多少依赖于根系 吸收 N 的数量, 可能遵循 投入-产出” ( Cost-benefit) 规律 (Eissenstat \& Yanai，1997)。土壤中有效 N 的含 量是有限的, 根系统中一些分枝细根因吸收作用很 快耗尽根际周围的有效 $\mathrm{N}$ 致使向这些根系投入的 $\mathrm{C}$ 减少 细根出现衰老而死亡 (Bloomfield et al ., 1996; Eissenstat \& Yanai , 1997)。根系只有不断生长, 才能 伸展到新的土壤空间去吸收有效 $\mathrm{N}$ (Burton et al. , $2000)$ 。因此, 土壤中的有效 $\mathrm{N}$ 与细根寿命有密切关 系(King et al ., 1999 ; Burton et al.,2000)。

一些野外林地试验表明,随着土壤有效 $\mathrm{N}$ 的增 加 细根寿命缩短，如栋树 (Quercus ilex) 林土壤有效 $\mathrm{N}$ 在 $143 \mathrm{~kg} \mathrm{hm}^{-2} \mathrm{a}^{-1}$ 时, 平均寿命 $167 \mathrm{~d}$, 有效 $\mathrm{N}$ 在 $133 \mathrm{~kg} \mathrm{hm}^{-2} \mathrm{a}^{-1}$ 和 $107 \mathrm{~kg} \mathrm{hm}^{-2} \mathrm{a}^{-1}$ 时, 平均寿命分 别为 $188 \mathrm{~d}$ 和 $301 \mathrm{~d}($ Aber et al . , 1985)。另一些试验 结果显示细根寿命随 $\mathrm{N}$ 有效性提高而延长, 如花旗 松( Pseudotsuga taxifolia) 林 (Keyes \& Grier , 1981)、糖 械 (Acer saccharum) 林 (Pregitzer et al ., 1993 ; Burton et $a l$. ,2000)。这些相悖结果引出细根寿命与 $\mathrm{N}$ 有效 性关系的一些假说 (Hendricks et al . , 1993 ;Eissenstat \& Yanai , 1997 ;Burton et al .,2000)。对于第一种情 况, 认为在有效性养分比较充足的土壤斑块，细根组 织中 $\mathrm{N}$ 浓度较高、呼吸增大 如果 $\mathrm{C}$ 的投入不能满足 呼吸消耗，导致细根寿命缩短（Burton et al.，1996; Black et al . , 1998) ,周转加速, 而贫瘦土壤有利于延 长细根的寿命 (Pregitzer et al . , 1995)。后一种情况， Burton 等 (2000)认为土壤有效 $\mathrm{N}$ 增加, 能够较长时间 地提供生长所需养分，只要有足够的 $\mathrm{C}$ 投入到具有 吸收功能的细根中去, 就可使细根寿命延长。Hendrick 和 Pregitzer(1996) 认为细根寿命与土壤养分有 效性可能呈正相关，也可能呈负相关，取决于植物种 类、器官或整个植物 $\mathrm{C}$ 平衡、有效养分在土壤中分布 的空间异质性等。他们还认为野外林地几年的施肥
试验导致细根寿命的延长或缩短仅是暂时现象，不 能代表肥沃土壤中 $\mathrm{N}$ 有效性对细根寿命的长期反 应。因此,Burton 等 (2000) 提出:不同树种或不同林 分类型中，每一个树种都有自身有效 $\mathrm{N}$ 的适应范围， 在这个范围内，细根寿命与土壤有效 $\mathrm{N}$ 的关系可能 呈正相关或负相关，但把这些对 $\mathrm{N}$ 有效性反应的不 同树种放在一起考察,总的规律是随着土壤有效 $\mathrm{N}$ 的增加 细根寿命缩短。这个假设目前还没有被试 验证明。如果该假设成立, 则对全球变化条件下预 测陆地生态系统的生产力具有重要意义, 因为土壤 $\mathrm{N}$ 的有效性受全球变化的影响而发生变化, 将引起 生态系统 $\mathrm{C}$ 分配格局的改变。

\section{4 细根寿命与土壤水分的有效性}

当土壤水分充足的时候，细根直径增粗，而干旱 条件下 细根直径减小, 寿命可能降低( Vogt \& Persson，1991）。由于水分还影响土壤养分的有效性，因 此, 水分对细根寿命的影响很难与养分的相互作用 分开 (Pregitzer et al. ,2000)。在干旱的立地上，细根 现存量较高, 周转率较快, 加速细根的死亡 (Santantonio \& Hermann , 1985)。C 的投入主要是促进细根的 生长，维持细根吸收水分的功能，尤其是促进细根向 深土层发展 (Hendrick \& Pregitzer , 1996 ; Joslin et al . , 2000)。但是, 长时间的干旱会引起树冠 C 同化率降 低, 常常不能满足细根生长的需要 细根因 $\mathrm{C}$ 的供应 不足而死亡。北美杨树 (Populus grandidentata)、红葪 栋( Quercus rubra)、和北美红枫 (Acer rubrum) 等林分 经过 20 40 d 灌溉后 细根寿命长达 82 90 d , 而不 灌溉的对照林分细根寿命仅为 $40 \mathrm{~d}$ 左右 (Pregitzer et $a l$. , 1993)。Comeau 和 Kimmins(1989)对湿润立地和 干旱立地生长的 70 年生小干松 (Pinus contorta var. latifolia) 研究表明, 干旱立地上细根 (直径 $<2 \mathrm{~mm}$ ) 和 小根 (直径 $<5 \mathrm{~mm}$ ) 生物量为 $6.4 \mathrm{t} \mathrm{hm}^{-2} \mathrm{a}^{-1}$, 年净 初级生产力为 $4.3 \sim 6.3 \mathrm{t} \mathrm{hm}^{-2} \mathrm{a}^{-1}$ (占总净初级生 产力的 $55 \% \sim 66 \%$ ), 而湿润立地上生物量为 $5.1 \mathrm{t}$ $\mathrm{hm}^{-2} \mathrm{a}^{-1}$, 年净初级生产力为 $4.5 \sim 5.5 \mathrm{t} \mathrm{hm}^{-2} \mathrm{a}^{-1}$ (占总净初级生产力的 $38 \% \sim 46 \%$ )。热带森林和蓝 桉 (Eucalyptus globulus) 人工林研究表明, 细根生长与 水分季节动态关系密切 细根生长高峰出现在雨季, 旱季生长减慢，死根量提高(Kaetterer et al ., 1995)。

对于土壤有效水分长期下降的影响,Joslin 等 (2000)认为该作用能够导致 : 1) 细根生物量、细根密 度、总伸长和净伸长增加;2)地下/地上比率, 特别是 细根生物量/叶生物量的比率增加；3)改变细根周转 速率；4)促进根系向下生长或增加深土层细根密度。 
这些假设在一些野外林分微根管的 4 年试验中得到 验证(Hendrick \& Pregitzer , 1996 ; Joslin et al . 2000)。 但是也有一些试验表明水分对细根生物量的现存量 影响不大(Tomlinson \& Anderson , 1998) ,但对地下/地 上比率影响显著, 干旱导致细根周转速率增加、引起 细根寿命缩短的假设在野外水分试验中都得到证实 (Joslin et al. , 2000)。但是水分对细根寿命的影响 与 $\mathrm{N}$ 的影响一样, 也具有两方面的特点, 不同树种细 根寿命对水分的反映存在明显差别, 需要多树种在 控制水分条件下试验，才能得出准确的结论。

\section{5 细根寿命与土壤温度}

Ryan (1991) 认为根系的寿命对土壤温度非常敏 感。Steele 等 (1997) 认为较高的土壤温度可增加细 根生产，促进细根的衰老和周转，缩短细根寿命。在 温带地区 ,Hendrick 和 Pregitzer(1993) 对密歇根州糖 槭林研究表明, 北部林分比南部林分细根寿命平均 延长 $75 \mathrm{~d}$ 。不同年均温的立地上细根的寿命差异与 土壤温度有关, 而同一立地上细根寿命的季节变化 也可能与土壤温度有关 (Burke \& Raynal , 1994)。在 温带气候条件下，晚春产生的细根寿命最短。如糖 槭从 6 月到 9 月产生的细根寿命从 $125 \mathrm{~d}$ 增加到 200 d(Hendrick \& Pregitzer , 1993)。Kosola 等 (1995) 对亚 热带树种的研究结果则与温带树种相反, 亚热带树 种秋天生产的细根寿命最短。在同一土壤剖面, 随 着土层加深, 温度下降, 土壤有效 $\mathrm{N}$ 降低, 细根的寿 命延长 (Burton et al. ,2000)。Gill 和 Jackson(2000) 总结了 190 个已发表资料的结果表明, 草地、灌木和 森林细根寿命与年均温具有明显的负相关, 从北方 到热带地区，细根周转提高，细根寿命显著降低，并 对这种关系给出 3 种可能的解释: 1 ) 高温能够提高 土壤养分 (尤其是 N) 的矿化速率;2) 维持根系的呼 吸随温度升高呈指数增长 3 ) 高温地区或非冻土区， 细根受病原菌侵染的可能性较大。Pregitzer 等 (2000) 也认为, 温度是限制土壤 $\mathrm{N}$ 有效性、根系呼 吸、C 消耗和土壤微生物活动的主要因子, 并由此解 释为什么热带地区森林细根周转较快, 温带和北方 森林细根周转较慢。

\section{6 细根寿命与土壤生物}

真菌、病原菌和土壤植食性动物是影响细根寿 命的主要土壤生物 (Eissenstat \& Yanai , 1997)。多数 树木的细根在不同程度上被菌根侵染。菌根的产生 不仅扩大细根的吸收表面积, 增加对 C 的消耗 (Peng et al. , 1993) , 而且对细根具有保护作用 (Black et $a l$ ， 1998），如内生菌根(VA) 和外生菌根 (EM) 通过 产生化学防御物质或对根尖的保护抵御病原菌对细 根的侵害 (Linderman，1994)、减少土壤动物的伤害 (Eissenstat et al ，2000)、增强忍受干旱胁迫的能力 (Espeleta \& Eissenstat, 1998) 等。大量实验证明菌根 能够延长根系的寿命, 降低根系的死亡率和周转速 率( Tierney \& Fahey , 2001)。例如, 没有菌根的细根 寿命仅有几周, 而侵染菌根的山毛榉 (Fagus grandifolia) 细根寿命接近 9 个月、太平洋银冷杉 (Abies amabilis ) 10～14 个月、欧洲云杉 (Picea excelsa) 48 个月 (Bloomfield et al. , 1996)。病原微生物也是影响细根 寿命的重要因子之一, 如根腐真菌可直接伤害根组 织和引起悾存的碳水化合物损失, 从而促进细根衰 亡 缩短了细根的寿命 (Shigo，1984)。

土壤植食性动物主要是地下昆虫和线虫 (Nematodes）,它们主要取食新生细根, 目的是获取碳水化 合物 (Eissenstat \& Yanai，1997)。Stevens 等 (2002)对 美国东南部 65 年生长叶松林的研究结果表明, 由土 壤植食性动物引起的细根死亡占死亡细根总量的 $21 \%$ ３7\%，导致一些新生细根很快消失。如果不 考虑土壤植食性动物的影响, 净生产力将被低估 $5 \%$ $\sim 10 \%$ 左右。在干旱和低温的条件下, 细根的寿命 较长, 除了维持需要的 $\mathrm{C}$ 较少外, 另一个原因是与同 样的环境下土壤植食性动物较少有关 (Eissenstat \& Yanai，1997)。此外, 植食性动物消耗少量的细根, 也能刺激细根生长, 但这取决于细根分布密度和地 下昆虫的种类 (Wardle，2002），土壤植食性动物对根 系的影响程度也决定了林木本身能在多大程度上控 制细根的寿命 (Yanai \& Eissenstat, 2002)。应当指出 的是, 土壤植食性动物对树木细根寿命和净初级生 产力的影响近几年才引起生态学界的注意, 目前还 没有结论性的解释, 但是作为影响细根周转和 $\mathrm{C}$ 消 耗的重要因子之一, 有必要进行深入研究 (Eissenstat et al . ,2000; Murray et al . 2002)。

\section{7 结 语}

国外关于细根的研究有近 30 年的历史, 对林木 细根寿命与细根周转的研究已经广泛开展, 尤其是 近 10 年来微根管的应用使得细根寿命与周转的研 究取得重要进展。但是, 关于细根寿命的控制机理 了解还很少, 其影响因素方面的研究仍然存在很多 争议。以往我国根系的研究主要包含在林分或群落 生物量研究之内, 近年来在细根生物量和周转方面 开展了一些研究(单建平等, 1993 ;廖利平等, 1995; 李 凌浩等, 1998 温达志等, 1999) ,但涉及影响细根周转 
和寿命的因素研究较少。无论在国内还是国外，细 根研究最大问题是结果的不确定性 (黄建辉等, 1999 张小全等,2000)，主要原因是研究方法缺乏一 致性与可比性、树种的多样性、立地条件差异、长期 试验与短期试验、单株树木研究结果到林分或生态 系统水平上尺度转换等问题。由此，建议我国对细 根寿命的研究应集中于以下几个方面 :1) C 的地下 分配可能是控制细根寿命的主要机理之一, 应着重 细根的周转过程与 $\mathrm{C}$ 分配的关系。2)土壤养分、水 分和温度对细根寿命有重要影响, 某一根段、整个根 系和林分水平上细根寿命与这些因子有何种关系? 需要大尺度和长期(包括不同气候区的)研究。3)细 根的寿命对资源有效性反应的研究得出不同的结 果, 这些研究多是短期试验。因此, 这种反应是否只 是暂时的现象，它与资源供应持续的时间有何关系？ 需要进行长期的研究去验证。4)细根本身的结构特 征(如直径、分枝位置等)与 $\mathrm{C}$ 的消耗有关，应进一步 了解 C 分配、有效资源获取与这些特征的关系。5) 由于细根的动态和土壤的空间异质性, 导致细根研 究结果的许多不确定性, 需要探讨有效和准确的方 法对细根寿命进行估计。

\section{参 考 文 献}

Aber, J. D., J. M. Melillo, K. J. Nadelhoffer, C. McClaugherty \& J. Pastor. 1985. Fine root turnover in forest ecosystems in relation to quality and form of nitrogen availability: a comparison of two methods. Oecologia, 66: $317 \sim 321$.

Anderson, C. P. 2003. Source-sink balance and carbon allocation below ground in plants exposed to ozone. New Phytologist, 157: $213 \sim 228$

Black, K. E., C. G. Harbron, M. Franklin, D. Atkinson \& J. E. Hooker. 1998. Differences in root longevity of some tree species. Tree Physiology, 18: $259 \sim 264$.

Bloomfield, J., K. A. Vogt \& P. M. Wargo. 1996. Tree root turnover and senescence. In: Waisel, Y., A. Eshel \& U. Kafkafi eds. Plant roots: the hidden half. 2nd ed. New York: Marcel Dekker. $363 \sim 382$.

Burke, M. K. \& D. J. Raynal. 1994. Fine root phenology, production and turnover in a northern hardwood forest ecosystem. Plant and Soil, 162: $135 \sim 146$.

Burton, A. J., K. S. Pregitzer \& R. L. Hendrick. 2000. Relationships between fine root dynamics and nitrogen availability in Michigan northern hardwood forests. Oecologia, 125: 389 399.

Burton, A. J., K. S. Pregitzer, G. P. Zogg \& D. R. Zak. 1996. Latitudinal variation in sugar maple fine root respiration. Canadian Journal of Forest Research, 26: $1761 \sim 1768$.

Chapin III, F. S., P. A. Matson \& H. A. Mooney. 2002. Principles of terrestrial ecosystem ecology. New York: Springer-Verlag. $123 \sim 147$

Comeau, P. G. \& J. P. Kimmins. 1989. Above- and belowground biomass and production of lodge pole pine on sites with differing soil moisture regimes. Canadian Journal of Forest Research, 19: $447 \sim 454$

Eissenstat, D. M. \& R. D. Yanai. 1997. The ecology of root lifespan. Advances in Ecological Research, 27: 1 60 .
Eissenstat, D. M. , C. E. Wells, R. D. Yanai \& J. L. Whitbeck. 2000. Building roots in a changing environment: implications for root longevity. New Phytologist, 147: $33 \sim 42$.

Espeleta, J. F. \& D. M. Eissenstat. 1998. Responses of citrus fine root to localized soil drying a comparison of seedlings with adult fruiting trees. Tree Physiology, 18:113 119 .

Farrar, J. F. \& D. L. Jones. 2000. The control of carbon acquisition by roots. New Phytologist, 147: $43 \sim 53$.

Fitter, A. H. 1985. Functional significance of root morphology and root system architecture. In: Fitter, A. H., D. Atkinson, D. J. Read \& M. B. Usher eds. Ecological interactions in soil: plants, microbes and animals. Special Bulletin Number 4 of the British Ecological Society. Oxford: Blackwell. 87 106 .

Gill, R. A. \& R. B. Jackson. 2000. Global patterns of root turnover for terrestrial ecosystems. New Phytologist, 147: 13 31 .

Gordon, W. S. \& R. B. Jackson. 2000. Nutrient concentrations of fine roots. Ecology, 81: $275 \sim 280$.

Gower, S. T., K. A. Vogt \& C. C. Grier. 1992. Carbon dynamics of Rocky mountain Douglas fir: influence of water and nutrient availability. Ecological Monographs, 62: $43 \sim 65$.

Haynes, B. E. \& S. T. Gower. 1995. Belowground carbon allocation in unfertilized and fertilized red pine plantations in northern Wisconsin. Tree Physiology, 15: $317 \sim 325$.

Hendrick, R. L. \& K. S. Pregitzer. 1993. Patterns of fine root mortality in two sugar maple forests. Nature, 361: 59 61.

Hendrick, R. L. \& K. S. Pregitzer. 1996. Temporal and depth-related patterns of fine root dynamics in northern hardwood forests. Journal of Ecology, 84: $167 \sim 176$.

Hendricks, J. J., J. D. Aber, K. J. Nadelhoffer \& R. D. Hallett. 2000. Nitrogen controls on fine root substrate quality in temperate forest ecosystems. Ecosystems, 3: $57 \sim 69$.

Hendricks, J. J., K. J. Nadelhoffer \& J. D. Aber. 1993. Assessing the role of fine roots in carbon and nitrogen cycling. Trees, 8 : $174 \sim 178$.

Hendricks, J. J. , K. J. Nadelhoffer \& J. D. Aber. 1997. A N ${ }^{15}$ tracer technique for assessing fine root production and mortality. Oecologia, 112: $300 \sim 304$.

Högberg, P., A. Nordgren, N. Buchmann, A. F. S. Taylor, A. Ekblad, M. N. Högberg, G. Nyberg, M. Ottosson-Löfvenius \& D. J. Read. 2001. Large-scale forest girdling shows that current photosynthesis drives soil respiration. Nature, 411: $789 \sim 792$.

Huang, J. H. (黄建辉), X. G. Han (韩兴国) \& L. Z. Chen (陈灵芝). 1999. Advances in the research of (fine) root biomass in forest ecosystems. Acta Ecologica Sinica (生态学 报), 19: 270 277. (in Chinese with English abstract)

Jackson, R. B., H. A. Mooney \& E. D. Schulze. 1997. A global budget for fine root biomass, surface area, and nutrient contents. Proceedings of the National Academy of Sciences, USA. 94: 7362 $\sim 7366$.

Joslin, J. D., M. H. Wolfe \& P. J. Hanson. 2000. Effects of altered water regimes on forest root systems. New Phytologist, 147: $117 \sim 129$.

Kaetterer, T., A. Fabiao, M. Madeira \& C. Ribeiro. 1995. Fine root dynamics, soil moisture and soil carbon content in Eucalyptus globules plantation under different irrigation and fertilization regimes. Forest Ecology and Management, 74: 1 12 .

Keyes, M. R. \& C. C. Grier. 1981. Above- and below-ground net productivity in 40-year-old Douglas fir stands on low and high productivity sites. Canadian Journal of Forest Research, 11: 599 605 .

King, J. S., K. S. Pregitzer \& D. R. Zak. 1999. Colonel variation in above- and below-ground growth responses of Populous tremuloides Michaux: influence of soil warming and nutrient availability. Plant and Soil, 217: 119 130 . 
Kosola, K. R., D. M. Eissenstat \& J. H. Graham. 1995. Root demography of mature citrus trees: the influence of Phytophthora nicotianae. Plant and Soil, 171: $283 \sim 288$.

Li, L. H. (李凌浩), P. Ling (林鹏) \& X. R. Xing (邢雪荣). 1998. Fine root biomass and production of Castanopsis eyrei forests in Wuyi Mountains. Chinese Journal of Applied Ecology (应用生态学报), 9: 337 340. (in Chinese with English abstract)

Liao, L. P. (廖利平)，C. Y. Chen (陈楚莹)，J. W. Zhang (张家武) \& H. Gao (高洪). 1995. Turnover of fine roots in pure and mixed Cunninghamia lanceolata and Michelia macclurei forests. Chinese Journal of Applied Ecology (应用生态学报), 6: $7 \sim 10$. (in Chinese with English abstract)

Linderman, R. G. 1994. Role of VAM fungi in biocontrol. In: Fleger, F. L. P. \& R. G. Linderman eds. Mycorrhizae and plant health. St. Paul. M N: American Phytopathological Press. $1 \sim 25$.

Majdi, H. 2001. Changes in fine root production and longevity in relation to water and nutrient availability in a Norway spruce stand in northern Sweden. Tree Physiology, 21: 1057 1061 .

Murray, P. J., L. A. Dawson \& S. J. Grayston. 2002. Influence of root herbivory on growth response and carbon assimilation by white clover plants. Applied Soil Ecology, 581: $1 \sim 9$.

Nadelhoffer, K. J. 2000. The potential effects of nitrogen deposition on fine root production in forest ecosystems. New Phytologist, 147: $131 \sim 139$.

Nadelhoffer, K. J., J. D. Aber \& J. M. Melillo. 1985. Fine root, net primary production, and soil nitrogen availability: a new hypothesis. Ecology, 66: $1377 \sim 1390$.

Norby, R. J. \& R. B. Jackson. 2000. Root dynamics and global change: seeking an ecosystem perspective. New Phytologist, 147: $3 \sim 12$.

Peng, S., D. M. Eissenstat, J. H. Graham, K. Williams \& N. C. Hodges. 1993. Growth depression of mycorrhizal citrus at high phosphorus supply: analysis of carbon costs. Plant Physiology, 101: $1063 \sim 1071$.

Pregitzer, K. S., D. R. Zak, P. S. Curtis, M. E. Kubiske, J. A. Teeri \& C. S. Vogel. 1995. Atmospheric $\mathrm{CO}_{2}$, soil nitrogen and turnover of fine roots. New Phytologist, 129: 579 585 .

Pregitzer, K. S., J. S. King, A. J. Burton \& S. E. Brown. 2000. Responses of tree fine roots to temperature. New Phytologist, 147: $105 \sim 115$.

Pregitzer, K. S., M. E. Kubiske, K. Y. Chui \& R. L. Hendrick. 1997. Relationships among root branch order, carbon, and nitrogen in four temperate species. Oecologia, 111: $302 \sim 308$.

Pregitzer, K. S. , R. L. Hendrick \& R. Fogel. 1993. The demography of fine roots in response to patches of water and nitrogen. New Phytologist, 125: $575 \sim 580$.

Pregitzer, K. S., J. L. DeForest, A. J. Burton, M. F. Allen, R. W. Ruess \& R. L. Hendrick. 2002. Fine root architecture of nine North American trees. Ecological Monographs, 72: 293 309.

Raich, J. W. \& K. J. Nadelhoffer. 1989. Below ground carbon allocation in forest ecosystems: global trends. Ecology, 70: 1346 1354 .

Ruess, R. W., R. L. Hendrick, A. J. Burton, K. S. Pregitzer, B. Sveinbjornsson, M. F. Allen \& G. E. Maurer. 2003. Coupling fine root dynamics with ecosystem carbon cycling in black spruce forests of interior Alaska. Ecological Monographs, 73: 643 $\sim 662$.

Ryan, M. G. 1991. Effects of climate change on plant respiration. Ecological Applications, 1: $157 \sim 167$.
Santantonio, D. \& R. K. Hermann. 1985. Standing crop, production, and turnover of fine roots on dry moderate and wet sites of mature Douglas fir in western Oregon. Annuals Deus Sciences Foresters, 42: $113 \sim 142$.

Schoettle, A. W. \& T. J. Fahey. 1994. Foliage and fine root longevity in pines. Ecological Bulletins, 43: 136 153 .

Shan, J. P. (单建平), D. L. Tao (陶大立), M. Wang (王水) \& S. D. Zhao (赵士洞). 1993. Fine roots turnover in a broadleaved Korean pine forest of Changbai Mountain. Chinese Journal of Applied Ecology (应用生态学报), 4: $241 \sim 245$. (in Chinese with English abstract)

Shigo, A. L. 1984. Compartmentalization: a conceptual framework for understanding how trees grow and defend themselves. Annual Review of Phytopathology, 22: $189 \sim 214$.

Steele, S. J., S. T. Gower, J. G. Vogel \& J. M. Norman. 1997. Root mass, net primary production and turnover in aspen, jack pine and black spruce forests in Saskatchewan and Manitoba, Canada. Tree Physiology, 17: $577 \sim 587$.

Stevens, G. N., R. H. Jones \& R. J. Mitchell. 2002. Rapid fine roots disappearance in a pine woodland: a substantial carbon flux to herbivores? Canadian Journal of Forest Research, 32: 2225 2230 .

Tierney, G. L. \& T. J. Fahey. 2001. Evaluating minirhizotron estimates of fine root longevity and production in the forest floor of a temperate broadleaf forest. Plant and Soil, 229: $167 \sim 176$.

Tomlinson, P. T. \& P. D. Anderson. 1998. Ontogeny affects response of northern red oak seedlings to elevated $\mathrm{CO}_{2}$ and water stress. II . Recent photosynthate distribution and growth. New Phytologist, 140: $493 \sim 504$.

Vogt, K. A. \& H. Persson. 1991. Measuring growth and development of roots. In: Lassoie, J. P. \& T. M. Hinckle eds. Techniques and approaches in forest tree ecophysiolgy. Boston: CRC Press. $477 \sim 501$.

Vogt, K. A., C. C. Grier, D. G. Sprugel \& D. G. Vogt. 1986. Overestimation of net root production: a real or imaginary problem? Ecology, 67: $577 \sim 579$.

Wardle, D. A. 2002. Communities and ecosystems: linking the aboveground and belowground components. Princeton: Princeton University Press. 58 136 .

Wells, C. E. \& D. M. Eissenstat. 2001. Marked differences in survivorship among apple roots of different diameters. Ecology, 82: $882 \sim 892$.

Wells, C. E., D. M. Glenn \& D. M. Eissenstat. 2002. Changes in the risk of fine-root mortality with age: a case study in peach, Prunus persica (Rosaceae). American Journal of Botany, 89: 79 $\sim 87$.

Wen, D. Z. (温达志), P. Wei (魏平), G. H. Kong (孔国 辉) \& W. H. Ye (叶万辉). 1999. Production and turnover rate of fine roots in two lower subtropical forest sites at Dinghushan. Acta Phytoecologica Sinica (植物生态学报), 23: $361 \sim 369$. (in Chinese with English abstract)

Yanai, R. D. \& D. M. Eissenstat. 2002. Coping with herbivores and pathogens: a model of optimal root turnover. Functional Ecology, 16: $865 \sim 869$.

Zhang, X. Q. (张小全) \& K. H. Wu (吴可红). 2001. Fine root production and turnover for forest ecosystems. Scientia Silvae Sinicae (林业科学), 37: 126 138. (in Chinese with English abstract)

Zhang, X. Q. (张小全), K. H. Wu (吴可红) \& D. Murach. 2000. A review of methods for fine root production and turnover of trees. Acta Ecologica Sinica (生态学报), 20: $875 \sim 883$. (in Chinese with English abstract) 